
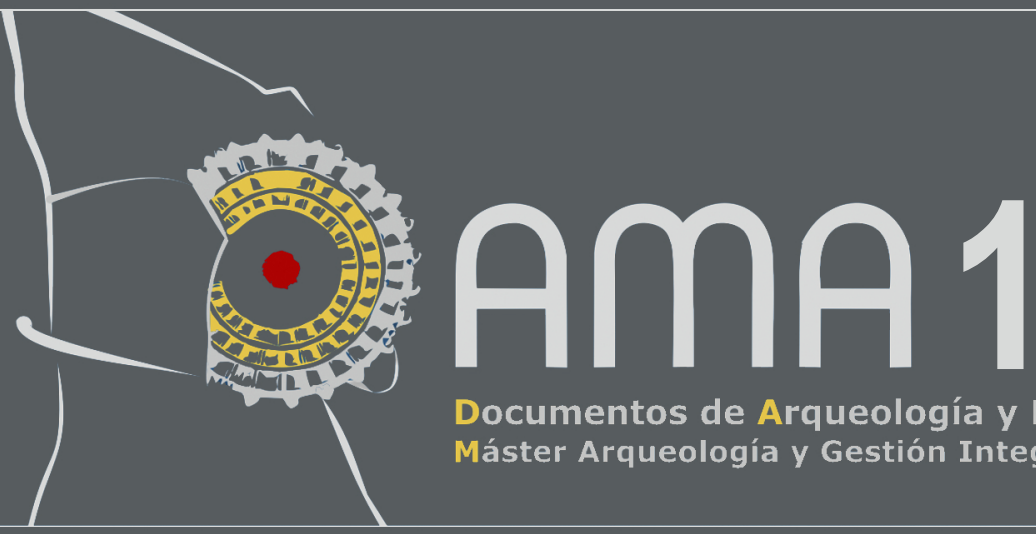

Documentos de Arqueología y Patrimonio Histórico

Máster Arqueología y Gestión Integral del Patrimonio de la Universidad de Alicante

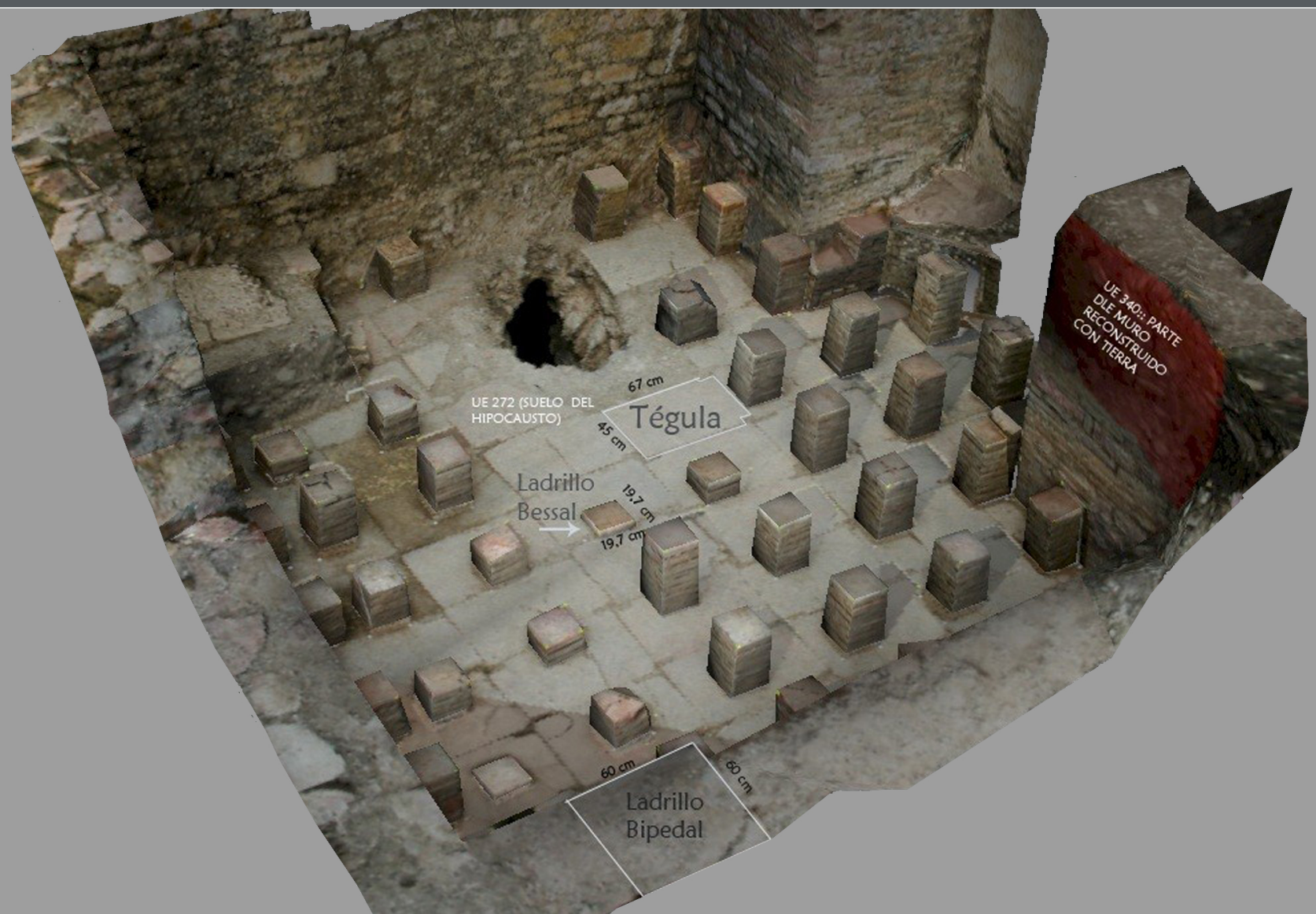

Documentos de Arqueología y Patrimonio Histórico Revista del Máster Universitario en Arqueología Profesional y Gestión integral del Patrimonio 



\section{DAMA 1}

\section{6}

DOCUMENTOS DE ARQUEOLOGÍA Y PATRIMONIO HISTÓRICO DEL MÁSTER UNIVERSITARIO EN ARQUEOLOGÍA PROFESIONAL Y GESTIÓN INTEGRAL DEL PATRIMONIO DE LA UNIVERSIDAD DE ALICANTE 


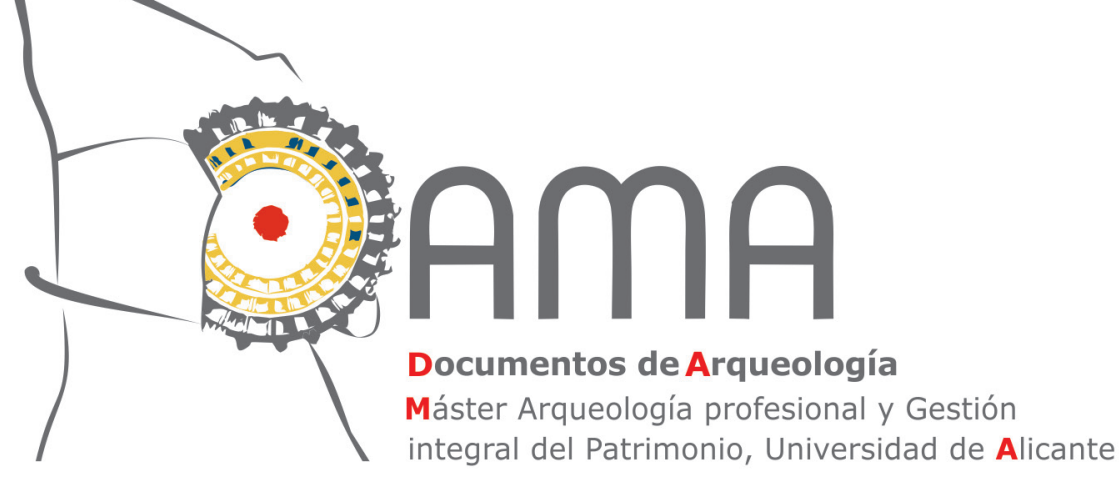

La revista electrónica DAMA. Documentos de Arqueología y Patrimonio Histórico surge como servicio para todos aquellos alumnos del Máster en Arqueología Profesional y Gestión Integral del Patrimonio de la Universidad de Alicante que se están iniciando en la investigación y cuya primera aportación a nuestra disciplina suele ser su Trabajo de Fin de Máster (TFM). Estos proyectos en muchos casos representan casi todo un curso de trabajo y esfuerzo, y con frecuencia quedan inéditos.

El objetivo de esta revista es ofrecer un medio que facilite la publicación de los resultados de sus TFM. La edición se presenta en versión digital y cuenta con su correspondiente ISSN. Se publica de forma anual en el sitio web de la Universidad de Alicante (http://web.ua.es/es/dama/) y en su repositorio (RUA). Los artículos publicados son descargables en formato PDF.

\section{Consejo de Redacción}

Directores (Coordinadores del Máster)

Carolina Doménech Belda

Fernando Prados Martínez

\section{Secretaria de Edición}

Julia Sarabia Bautista

\section{Vocales}

Los miembros de la Comisión Académica del Máster Universitario en Arqueología Profesional y Gestión del Patrimonio (http://dprha.ua.es/es/magip/comision-academica.html)

\section{Edita}

Máster Universitario en Arqueología Profesional y Gestión Integral del Patrimonio Departamento de Prehistoria, Arqueología, Historia Antigua, Filología Griega y Filología Latina

Facultad de Filosofía y Letras II

Universidad de Alicante

Ctra San Vicente del Raspeig s/n

E-03690 San Vicente del Raspeig (Alicante)

Web:http://dprha.ua.es

Teléfono: (+34) 965903663

Fax: (+34) 965903823

E-mail: revista.dama@ua.es

\section{ISSN}

$2530-2345$

\section{Portada}

Modelo fotogramétrico de un hypocaustum romano realizado por Ana Charquero 
EDITORIAL

Coordinación del máster

ENTREVISTA A SOLVEIG NORDSTRÖM: UNA MIRADA HACIA EL PASADO DE LA ESCUERA

Raúl Berenguer González ..

\section{ARQUEOLOGÍA Y MÉTODO}

EL ESTUDIO DE LOS MATERIALES CONSTRUCTIVOS DE TIERRA DEL CABEZO DEL POLOVAR (VILLENA, ALICANTE): APORTACIÓN A LAS FORMAS CONSTRUCTIVAS DE DOS PEQUEÑAS EDIFICACIONES CAMPESINAS DE LA EDAD DEL BRONCE EN EL LEVANTE PENINSULAR

María Pastor Quiles

VIVIENDAS POSTALAYÓTICAS: UNA APROXIMACIÓN A LOS ESPACIOS DOMÉSTICOS EN EL ARCHIPIÉLAGO BALEAR (550-123 ANE)

Octavio Torres Gomáriz

LAS FLOTAS DE GUARNICIÓN ROMANA EN LAS COSTAS DE HISPANIA A PARTIR DE LAS FUENTES EPIGRÁFICAS

Sergio Lledó Ramírez

UNA APROXIMACIÓN A LA RECONSTRUCCIÓN DEL PAISAJE HISTÓRICO: EL PROYECTO L'ALMISSERÀ Diana López Arroyo

NUEVAS PERSPECTIVAS PARA EL ESTUDIO ARQUEOLÓGICO DEL POBLAMIENTO RURAL MEDIEVAL EN ASPE (ALICANTE): HUERTAS Y ALQUERÍAS JUNTO AL RÍO TARAFA

Felipe Mejías López

ARQUEOLOGÍA DE LAS GUERRAS CARLISTAS

Iván Roldán Vergarachea

LA CERÁMICA DE USO ARQUITECTÓNICO EN NOVELDA: LA AZULEJERÍA DE FINALES DEL SIGLO XIX Y PRINCIPIOS DEL XX

Natalia Sala Pérez

PRÁCTICA Y USOS DE LA FOTOGRAMETRÍA DIGITAL EN ARQUEOLOGÍA

Ana $M^{a}$ Charquero Ballester.

\section{GESTIÓN Y PUESTA EN VALOR DEL PATRIMONIO}

ARQUEÓLOGOS CON DISCAPACIDAD. ARQUEOLOGÍA INCLUSIVA

Ana Samaniego Espinosa

"LES COVES DELS PESCADORS DE EL CAMPELLO": PROPUESTA DE RECUPERACIÓN, PUESTA EN VALOR Y USO PÚBLICO DE UN PATRIMONIO OLVIDADO

Ana Isabel Castro Carbonell .

LA COLONIA DE SANTA EULALIA. ESTUDIO Y PROPUESTA DE RECUPERACIÓN

Héctor de Arriba González

PROYECTO DE PUESTA EN VALOR DE LOS RESTOS DE LA GUERRA CIVIL EN LA CIUDAD DE ALICANTE

Leticia Victoria González Chouciño

ANTEPROYECTO MUSEOGRÁFICO DEL MUSEO DE HISTORIA DE SAX

Alberto Ochoa García

EL CONGRESO DE MÁSTER: UNA PROPUESTA DIDÁCTICA DEL MÁSTER DE ARQUEOLOGÍA DE LA UNIVERSIDAD DE ALICANTE

Ignasi Grau Mira, Sonia Gutiérrez Lloret, Carolina Doménech Belda, Julia Sarabia Bautista 



\title{
VIVIENDAS POSTALAYÓTICAS: UNA APROXIMACIÓN A LOS ESPACIOS DOMÉSTICOS EN EL ARCHIPIÉLAGO BALEAR (550-123 ANE)
}

\author{
Octavio Torres Gomariz
}

\section{RESUMEN}

Este trabajo tiene por objetivo realizar una aproximación al estudio de los espacios domésticos durante el período Postalayótico (550-123 ANE) en las Islas Baleares como medio para entender y analizar los grupos domésticos que constituían estas sociedades insulares. Para ello, se trazará la metodología seguida y las hipótesis de partida, finalizando con una hipótesis preliminar sobre la existencia de un modelo doméstico característico de estos momentos.

Palabras clave: Arquitectura doméstica, Postalayótico, Vivienda, Islas Baleares

\section{ABSTRACT}

This paper aims to make an approach to the study of domestic space during the Postalayotic period (550-123 ANE) in the Balearic Islands as a means to understand and analyze domestic groups constituting these island societies. To do this, the methodology and assumptions will be drawn starting, ending with a preliminary hypothesis on the existence of a domestic model.

Key words: Domestic architecture, Postalayotic, House, Balearic Islands 


\section{INTRODUCCIÓN: EL ARCHIPIÉLAGO BALEAR BAJO ÓRBITA PÚNICA}

A mediados del siglo VI ANE, las comunidades humanas que habitaban las Islas Baleares experimentaron una serie de cambios transversales, observables a través de la materialidad del registro arqueológico. Son muchas las razones que se han esgrimido para explicar estos fenómenos de transformación de la sociedad balear. Una de ellas es la inclusión efectiva del archipiélago en el área de control político y económico púnico a mediados del siglo VI ANE, que vendría auspiciada por la cercana colonia fenicio-púnica de 'ybshm/Ebusus (Ibiza).

A pesar de encontrarse en una posición estratégica en el Mediterráneo central, óptima para dicha órbita política y comercial, no se ha estudiado de forma exhaustiva el contacto e interacción de la sociedad insular con las culturas levantinas y norteafricanas que recorrieron el mar a lo largo del I Milenio, salvo en el caso de Mallorca, donde sí se ha mantenido un interés constante por este asunto (Guerrero, 1984 y posteriores). En las Gimnesias ha primado historiográficamente el conocimiento de épocas anteriores, las denominadas Naviforme y Talayótica. Ello ha conllevado la conformación de un amplio período historiográfico, el Postalayótico, de dilatada cronología (siglos VI - II ANE), pero desconocido en gran medida. Este trabajo nace con la vocación de llamar la atención sobre este momento, profundizando en su problemática inherente y acentuando la investigación desde la perspectiva de la interacción entre las comunidades postalayóticas insulares y el ámbito púnico.

No obstante, dado el amplio espectro del periodo, ha sido preciso seleccionar un ámbito de estudio en el que centrar la investigación. Diversas publicaciones han abordado aspectos concretos y/o generales del periodo (algunos de los más recientes, Palomar, 2005; Guerrero et al., 2006; Lull et al., 2008; Calvo y Guerrero, 2011; entre otros). Por tanto, finalmente se ha escogido un aspecto transversal, la unidad básica de cualquier sociedad: sus grupos domésticos y su correspondiente espacio de hábitat, las casas. Las viviendas postalayóticas han sido objeto de análisis en diversos casos, pero pocas veces de forma exhaustiva y sistematizada. Por ello, se hace necesario un compendio sintético de esta arquitectura doméstica que ilustre los cambios acontecidos entre los siglos VI - II ANE. Además, si se produjo esa inclusión efectiva de las Gimnesias en el ámbito fenicio-púnico, ésta debió dejar indudablemente su impronta también en los ámbitos domésticos y las personas que en ellos habitan.

Cabe destacar finalmente que este trabajo se inserta en un proyecto de investigación de la Universidad de Alicante: Proyecto Modular. Arquitectura fenicio-púnica dirigido por el Dr. Fernando Prados Martínez. Éste tiene como principal objeto de estudio el mundo fenicio-púnico desde sus manifestaciones arquitectónicas, con el objetivo de la caracterización de su cultura arquitectónica en diversos escenarios y a través del análisis de todas sus materializaciones, sean defensivas, domésticas, funerarias o de culto.

\section{ESPACIOS DOMÉSTICOS A TRAVÉS DE LA GRAMÁTICA DE LA CASA}

Partiendo de estos planteamientos, es preciso definir una metodología de estudio cuya finalidad sea la resolución de la problemática que origina este trabajo. En este caso, esta metodología se ha denominado gramática de la casa, nombre tomado prestado de la obra de S. Gutiérrez Lloret (2012), en cuyo trabajo homónimo reflexiona sobre los métodos empleados para la caracterización de espacios domésticos medievales en la península Ibérica. En él, la autora apuesta por la aplicación de una metodología a modo de gramática, entendiendo así que las viviendas constan de formas básicas materiales, relaciones entre dependencias, y finalmente un significado general compuesto por la propia unión de los elementos anteriores. De esta forma, la casa se convierte en un escenario privilegiado de observación, pues es un producto social que crea y reproduce la propia sociedad, en tanto que actúa como medio de expresión y transmisión de conductas y comportamientos (Gutiérrez, 2012, p. 139). 
Por tanto, para su estudio e interpretación, se precisa de un análisis complejo para acometer a posteriori el estudio de su articulación interna y organización. La unión de todas ellas permitirá la inferencia de significados culturales sobre el espacio doméstico construido y su uso. Adaptando los planteamientos explicitados por Gutiérrez (2012, pp. 139-140) y partiendo de estas premisas, la propuesta metodológica que se propone consta de tres ejes principales, cuya combinación es conditio sine qua non para obtener una visión íntegra y completa del objeto de estudio.

En primer lugar, el estudio de la morfología atiende a la forma de las viviendas, esto es a sus elementos constitutivos, las técnicas empleadas (aparejos, técnicas, elementos empleados, etc.) en su construcción, la planta obtenida como resultado de su ejecución, así como sus modificaciones y transformaciones posteriores. Este primer elemento, el más corriente en los estudios arquitectónicos desde perspectivas arqueológicas, es la base de la metodología empleada y será la parte que se aborde en este trabajo. Tiene un marcado carácter descriptivo, analítico y funcionalista, generalmente empleado para establecer taxonomías y tipologías.

En segundo lugar, la sintaxis entre las estructuras documentadas, es decir, las relaciones espaciales que tienen unas dependencias con otras a través de diversos vectores de análisis (accesibilidad, visibilidad, etc.). Todo ello se obtiene mediante la aplicación de herramientas específicas, procedentes en origen de estudios arquitectónicos o de urbanismo geográfico, destinadas al análisis espacial de las viviendas (Hillier y Hanson, 1984; Blanton, 1994). Finalmente, con los datos generados de los elementos anteriores, llegamos a la semiótica, la interpretación y significado dados a las relaciones entre el espacio construido y la estructura social que los concibe. Todo ello acompañado del estudio exhaustivo de las expresiones sociales materializadas a través de la arquitectura doméstica, así como del uso social del propio entorno construido.

Esta metodología, aplicada al objeto de estudio y a su contexto espacial y temporal que aquí concierne, se traduce en los siguientes pasos. Se ha partido de la reunión de toda la información necesaria para caracterizar morfológicamente la arquitectura doméstica del Postalayótico balear. El análisis arquitectónico y descriptivo exhaustivo se vertebra en tres ejes básicos: los materiales empleados, las técnicas de construcción identificadas y las plantas arquitectónicas generadas. Todo ello configura un corpus descriptivo, sistematizado y sintético, base necesaria para cualquier estudio posterior, como materialización de un conocimiento amplio del registro arqueológico para poder aplicar otras herramientas de análisis e interpretar finalmente esos datos.

Seguidamente se ha ejecutado el estudio pormenorizado de la morfología de dos casos de estudio sobre los que se han aplicado a posteriori las herramientas de sintaxis espacial.Éstas han proporcionado una serie de datos cuantitativos que, sumados a los de carácter descriptivo y característico, aportan nuevas vías de lectura de los espacios domésticos. La elección únicamente de dos casos responde a las limitaciones que presentan este tipo de herramientas de análisis espacial (Bermejo, 2009) y la disponibilidad de datos al respecto. Sin embargo, para alcanzar unos resultados contrastables, válidos a nivel de investigación general y comparada, es necesario aplicar esta metodología a un número mucho mayor y más representativo, de casos de estudio, por lo que no se desarrollarán aquí sus resultados provisionales.

Finalmente, se deben interpretar todos los datos observados y generados, como paso último de cualquier investigación, creando un discurso histórico que dé explicación a las viviendas postalayóticas per se y lo que suponen a nivel ilustrativo sobre la sociedad que las habitan. El objetivo es por ende transformar toda la información generada en características que nos hablen de los grupos domésticos que habitaban las casas analizadas. ¿Cómo? Mediante la lectura funcional de los espacios domésticos, la definición de particularidades morfológicas y sintácticas, que llevan implícitas rasgos sobre las comunidades que las han construido, estableciendo modelos comparativos y planteando hipótesis 
aproximativas sobre la estructura y el carácter de los grupos domésticos. Para todo ello se deben implementar todos los conocimientos sobre el resto de ámbitos de la cultura postalayótica, como son el territorial, funerario, cultura material, etc., pues cualquier lectura interpretativa que se realice debe enmarcarse en su correspondiente contexto histórico.

\section{EL POSTALAYÓTICO BALEAR (550-123 ANE): VICISITUDES DE UNA ÉPOCA DE TRANSFORMACIONES}

Lo que conocemos actualmente como Postalayótico esconde una realidad compleja, incluso confusa a día de hoy. Las características monumentales del período previo, el Talayótico, hicieron de él prácticamente el eje fundamental sobre el que pivotaba toda la Prehistoria del archipiélago, siendo referencia para los períodos previos y posteriores. El Postalayótico es la última etapa de la Protohistoria en ambas islas (Mallorca y Menorca), previa a la conquista romana que tiene el año 123 ANE como consenso general y corresponde a la conquista de Mallorca por el general romano Q. Cecilio Metelo, pese al reconocimiento generalizado de que esta última fecha no refleja cambios sustanciales en el devenir insular (Palomar, 2005, entre otros) hasta tiempos posteriores.

El punto de inflexión, por ser el más ilustrativo de la ruptura respecto a la etapa anterior, es el fin de la construcción de talayots en las islas y su amortización estructural en la mayoría de los casos. Estos recintos, característicos de la fase anterior, dejan de erigirse en torno al 700-650 ANE (Micó, 2005). Teniendo presente el papel central que ejercían en la configuración de los poblados talayóticos, principalmente en cuanto a la articulación del territorio y su control estratégico; y la importancia social que tenían por ende estas construcciones para las comunidades que las erigieron (Guerrero et al., 2006, pp. 21-22), este hecho refleja indudablemente un profundo cambio político y social en el seno de las sociedades baleáricas.

Al mismo tiempo que cesa la construcción de los talayots, los poblados inician diversos procesos de amurallamiento. Asentamientos como Son Fornés (Montuïri, Mallorca) o Trepucó (Maó, Menorca) alzan sus murallas delimitando sus respectivos núcleos urbanos (Hernández-Gasch y AramburuZabala, 2005). De forma paralela, se detectan numerosos casos de ampliación de dichos núcleos, ya existentes, o la erección de nuevas barriadas, así como de pequeños asentamientos costeros (Calvo, 2009, p. 64). Este fenómeno, que muestra por sí mismo un aumento dirigido del hábitat, ha sido interpretado historiográficamente como una nueva concepción del dominio del espacio inmediato, que en estos momentos emplea la propia antropización del territorio, u otras formas de significación, como la fortificación de los poblados, y no mediante la semantización arquitectónica y visual mediante turriformes (Calvo, 2009, p. 66).

En cuanto a los ámbitos funerarios y religiosos, la perduración de ritos propiamente talayóticos, como los enterramientos colectivos en cal, viene acompañada de la consolidación progresiva de la individualización mediante los contenedores mortuorios. Este rasgo expone la voluntad de las comunidades humanas de esta época de diferenciarse socialmente a través de la muerte, hecho no detectado hasta el Postalayótico (Palomar, 2005, p. 39). Por otra parte, otro de los grandes cambios de esta fase postalayótica es la aparición de los denominados santuarios mallorquines y recintos de taula menorquines (Guerrero et al., 2006). La mera aparición de estos conjuntos arquitectónicos denota transformaciones notorias en el plano simbólico e ideológico de estas comunidades, al ocupar en muchas ocasiones la importancia espacial que antes tenían los turriformes.

De forma paralela a todos estos fenómenos, uno de los rasgos que afectan de forma transversal a la vida cotidiana de estas comunidades es la intensificación de los intercambios con el ámbito púnico, canalizados especialmente a través de la colonia púnica de 'ybshm/Ebusus. Este aumento de los intercambios comerciales se enmarca en la expansión del estado colonial cartaginés a lo largo y 
ancho del Mediterráneo, aunque con especial incidencia en las zonas central y occidental, como se aprecia en las vecinas islas de Cerdeña y Sicilia, donde se detectan procesos de contacto paralelos (recogidos, por ejemplo, en Delgado y Ferrer, 2007). Será entre los siglos V-IV ANE cuando este auge alcance la estandarización comercial entre ambos agentes, que conllevó, entre otros aspectos, una importante llegada de productos púnico-ebusitanos a las Gimnesias.

Por otra parte, es necesario prestar atención al desarrollo de la isla ibicenca en estos momentos. Siguiendo un proceso de evolución interna que arranca con la fundación de la colonia fenicia de Sa Caleta (Ramón, 2007), a finales del siglo V y a lo largo del siglo IV ANE, 'ybshm/Ebusus se consolida como una ciudad-estado púnica (Costa y Fernández, 2006, p. 102). Concentraba toda la actividad política, económica y administrativa, al tiempo que era el núcleo de referencia de toda la población dispersa por la isla. Consolidando el modelo productivo comercial iniciado, el territorio insular púnico-ebusitano se convierte en un centro exportador de productos, articulando su cadena de producción en dos ejes: la creación de una densa malla de asentamientos agrícolas (llamados historiográficamente granjas púnicas); y el desarrollo de una industria de transformación de productos agrarios y de alfarería, tanto de envases de transporte, vajilla común y de mesa, y diversas artesanías, todo ello en cualquier caso con clara vocación de una economía agrícola, pero especialmente comercial exportadora que hizo del Mediterráneo occidental su espacio de comercio hegemónico.

El incremento cuantitativo y cualitativo de los contactos entre púnicos y las comunidades baleáricas influirá de manera determinante en la estructura de estas últimas, configurando lo que hoy conocemos como sociedad postalayótica. Este fenómeno no es un caso aislado ni particular, sino que se viene produciendo de forma recurrente con el contacto entre culturas indígenas y el ámbito fenicio-púnico (como se observa en otros contextos insulares, como Cerdeña y Sicilia, pero también en la península Ibérica o el norte de África). En este caso, la llegada de dichos agentes y su interactuación con las comunidades autóctonas supone una serie de cambios y alteraciones, diferentes en tiempos y lugares, pero que afectan de forma transversal a ambas sociedades, generando culturas mestizas o hibridas (Prados et al., 2015).

\section{ESPACIOS, FORMAS Y TÉCNICAS DE LAS CASAS POSTALAYÓTICAS}

Como se ha explicitado anteriormente, la base del trabajo es la creación de un corpus documental donde figuren todos los ámbitos domésticos identificados hasta el momento en las Gimnesias e Ibiza. Son tres los ejes que articulan el compendio de viviendas recogidas: los materiales y herramientas empleados en su erección, las técnicas constructivas identificadas y las formas resultantes de la construcción, esto es, dependencias, plantas, etc. A nivel metodológico básico, la base empírica de este corpus consiste en la extracción y recopilación de los datos necesarios mediante la búsqueda bibliográfica y/o la observación in situ. Todo ello ha quedado reflejado en fichas creadas expresamente para esta tarea, desarrolladas en el marco del Proyecto Modular (Prados et al., 2015), y finalmente se han registrado en una base de datos.

Este procedimiento no es novedoso, pues ya los tratadistas arquitectónicos clásicos, como B. Zevi, apuntaron que el primer paso para abordar el estudio arquitectónico es partir de un catálogo sistematizado (1978, p. 21). A modo de ejemplo, en el campo de la arqueología de nuestro país, contamos con una larga tradición de estudios arquitectónicos basados fundamentalmente en la caracterización morfológica de las estructuras arquitectónicas (por ejemplo Plantalamor, 1991; Abad y Sala, 1993; 2007; Belarte, 1997; o Moret, 2002) para tratar de inferir posteriormente consideraciones de carácter socio-cultural.

La base teórica que da sentido a esta fase metodológica entiende que aprehender la codificación de los ámbitos domésticos permitirá dilucidar cuestiones a gran escala de índole política, social, 
económica e ideológica. En este caso además, el análisis detallado de las casas nos informará del grado de aceptación, implantación e imbricación de nuevas normas sociales, producto de una situación distinta a la anterior, como es la presencia de factores exógenos (fenicio-púnicos) en contacto con las comunidades autóctonas. En este aspecto, se ha incorporado el concepto de cultura arquitectónica (Prados, 2003), entendido como todas las formas arquitectónicas que configuran el paisaje urbano y político de una sociedad en particular, reconocidas por tanto como propias, identitarias, por ellos mismos y ante sus coetáneos. De esta forma, se conciben las tradiciones constructivas como elementos dinámicos, tendentes a compartir y adoptar técnicas o fórmulas arquitectónicas en el marco de un contacto entre diversos agentes, superando las posibles barreras ideosincráticas, y asimilando conceptos urbanísticos o morfológicos en general que conllevan el cambio de los patrones sociales previos.

Sintetizando la información recabada, en el caso de Mallorca se han analizado numerosos ámbitos domésticos identificados a lo largo de diversos yacimientos. Entre ellos se encuentran la Estancia 19 de S'Illot (Sant Llorenç des Cardassar) (Frey, 1968; Krause, 1978); las múltiples viviendas de Ses Païsses (Artà) (Aramburu-Zabala y Hernández-Gasch, 2005; entre otros); algunos espacios de Capocorb Vell (Llucmajor) (Font Obrador, 1970) reestudiados recientemente (Hernández-Gasch, 2010); las viviendas postalayóticas de Son Fornés (Montuïri) (Palomar, 2005; Lull et al., 2008; etc.); el Edificio Alfa de Puig Morter de Son Ferragut (Sineu) (Castro et al., 2033); el Turó de ses Beies (Santa Ponça) (Camps y Vallespir, 1998) y finalmente también se ha prestado atención a las denominadas factorías costeras púnico-ebusitanas de Na Guardis (Ses Salines), Na Galera (Palma) y Es Trenc (Campos), estudiadas de forma exhaustiva por Guerrero (1997; 2000; 2003, etc.).

\section{Sin tiempo ni espacio para comentar cada uno de los espacios domésticos} analizados, sí se abordará una caracterización general. Aunque pervivan algunos ejemplos de plantas arriñonadas (como la Estancia 19 de S'Illot), las casas postalayóticas mallorquinas tienden poco a poco hacia plantas eminentemente rectangulares o cuadrangulares (Fig. 1). $\mathrm{Su}$ construcción presenta un panorama variado, yendo de la mampostería bien escuadrada de mediano y pequeño tamaño (S'Illot o Capocorb Vell), a la progresiva hegemonía de un aparejo ciclópeo tripartito (paramento exterior de grandes bloques, relleno de tierra y ripio

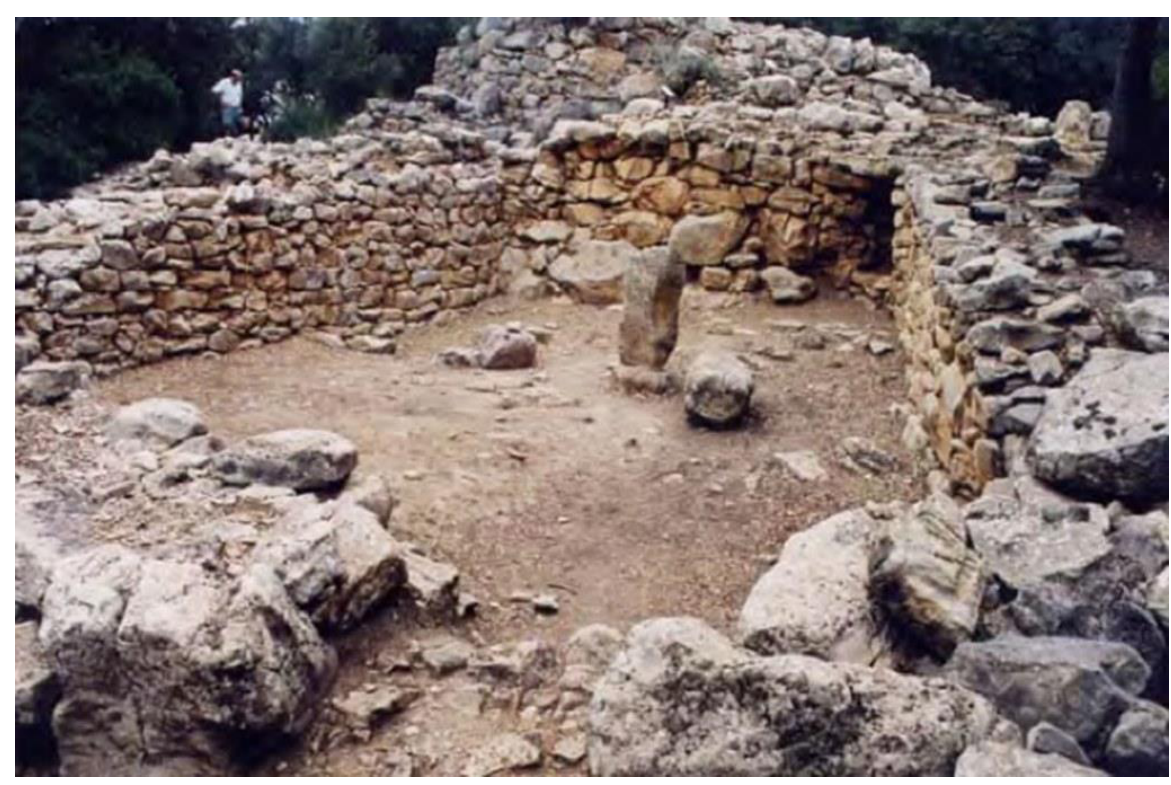

Fig. 1. Estancia R9 de Ses Païsses en la que se observa la planta cuadrangular (Fuente: Aramburu-Zabala y Hernández-Gasch, 2005, p. 40). y paramento interno de mampostería pequeña a tizón), como ilustran las viviendas de Son Fornés o Ses Païsses (Fig. 2). Por otra parte, destaca la presencia recurrente de columnas exentas, adosadas o insertas en los muros perimetrales y/o medianeros. Los materiales de construcción proceden generalmente del entorno inmediato de los yacimientos. De los pocos casos que se han detectado, podemos inferir cubiertas de elementos vegetales trabados con tierra (Edificio 25 de Ses Païsses). En cuanto a los pavimentos, generalmente se trata de la roca madre, aunque en algunos casos podemos encontrar enlosados (HTP1 de Son Fornés) o incluso morteros preparados (Turó de ses Beies). 
De forma mayoritaria, las viviendas presentan tres espacios: uno más grande situado generalmente al norte y siempre el primero al que se accede; y dos en la parte posterior de la casa, divididos por sendos muros que intersecan, realizados generalmente de mampostería pequeña bien aparejada en hiladas. El espacio más grande es el que mayor número de infraestructuras presenta, desde cisternas y hogares hasta bancos corridos y enlosados, acompañados siempre de instrumental lítico (molinos, morteros, etc.). Las posteriores son más heterogéneas, abundando la cerámica a mano local y las importaciones, así como herramientas de trabajo y procesado de alimentos (los restos vegetales también abundan). Las características descritas permiten formular un modelo de casa concreto compuesto por un espacio interior más grande donde se realizan la mayor parte de las tareas domésticas de carácter productivo (facilitando la salida de humos, recogida de aguas, etc.); mientras que en las posteriores, más pequeñas, se desarrollan otras actividades concretas o se destinan al almacenamiento o descanso del grupo residente.

Mientras tanto, el panorama observable en la vecina isla de Menorca dista notablemente (al menos a priori) de lo que se ha visto hasta ahora. En la isla menorquina se consolida un patrón constructivo de viviendas, una suerte de casa-tipo. Los denominados cercles menorquines, o círculos de hábitat, han sido el resultado de un largo proceso historiográfico, siendo definidos y caracterizados de forma exhaustiva por primera vez por la arqueóloga M. L. Serra Belabre, basándose en sus excavaciones durante los años 50 y 60 del siglo pasado en los yacimientos de Sant Vicenç d’Alcaidús (Alaior) (Serra, $1961 ; 1962 ; 1964 ;$ etc.).

Estos círculos tienen plantas de morfología oscilante entre el círculo y la herradura, siendo en este último caso la fachada generalmente recta y el resto de tendencia absidal. Su construcción en todos los

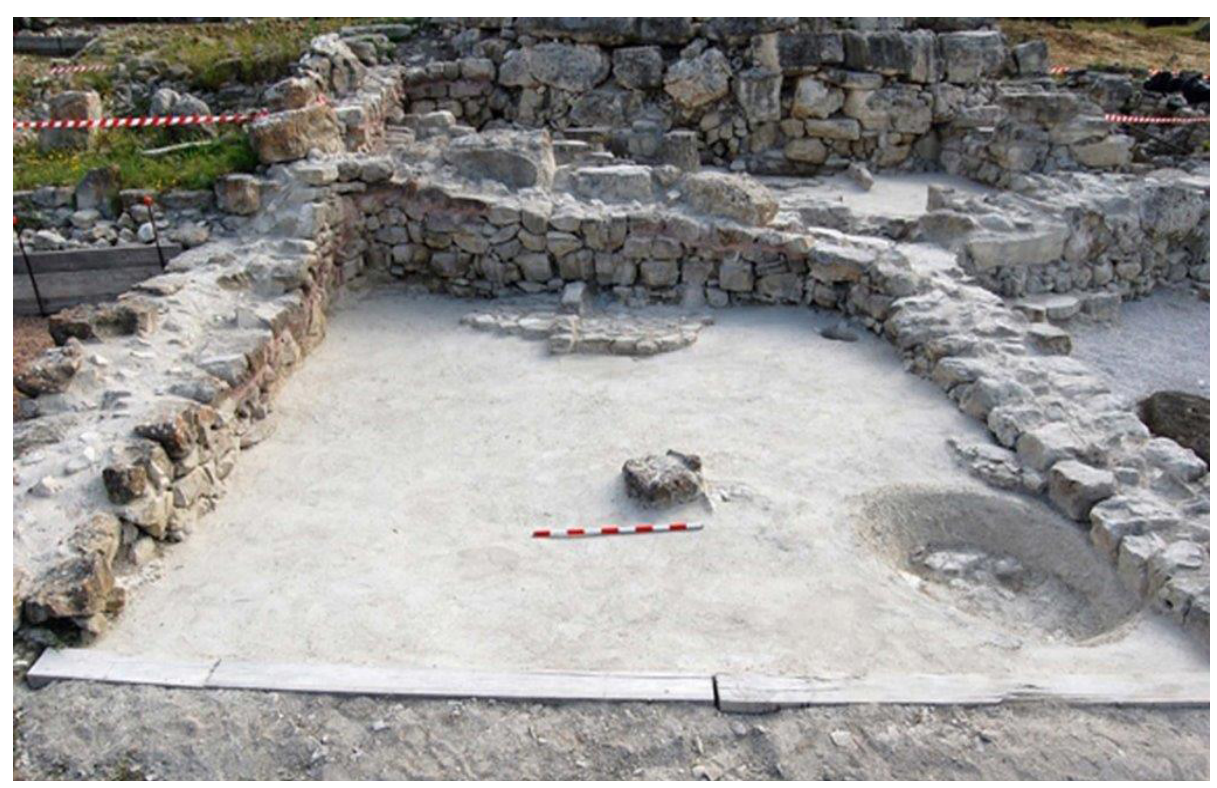

Fig. 2. Vivienda HTP1 de Son Fornés (Fuente: Museu Arqueològic de Son Fornés). encontramos una mampostería de bloques cuadrangulares, escuadrados y desbastados, jalonados con pilastras adosadas o intestadas (generando un opus africanum poco convencional).

El espacio más característico de estas viviendas es el patio central (Figs. 3 y 4). Ya M. L. Serra lo identificó como tal, atendiendo principalmente a su función articuladora del espacio doméstico, pues a través de él se accede a todas y cada una de las estancias que conforman la vivienda (Serra, 1965). En el patio, formando un pequeño ámbito segregado de planta semicircular, se encuentra generalmente 

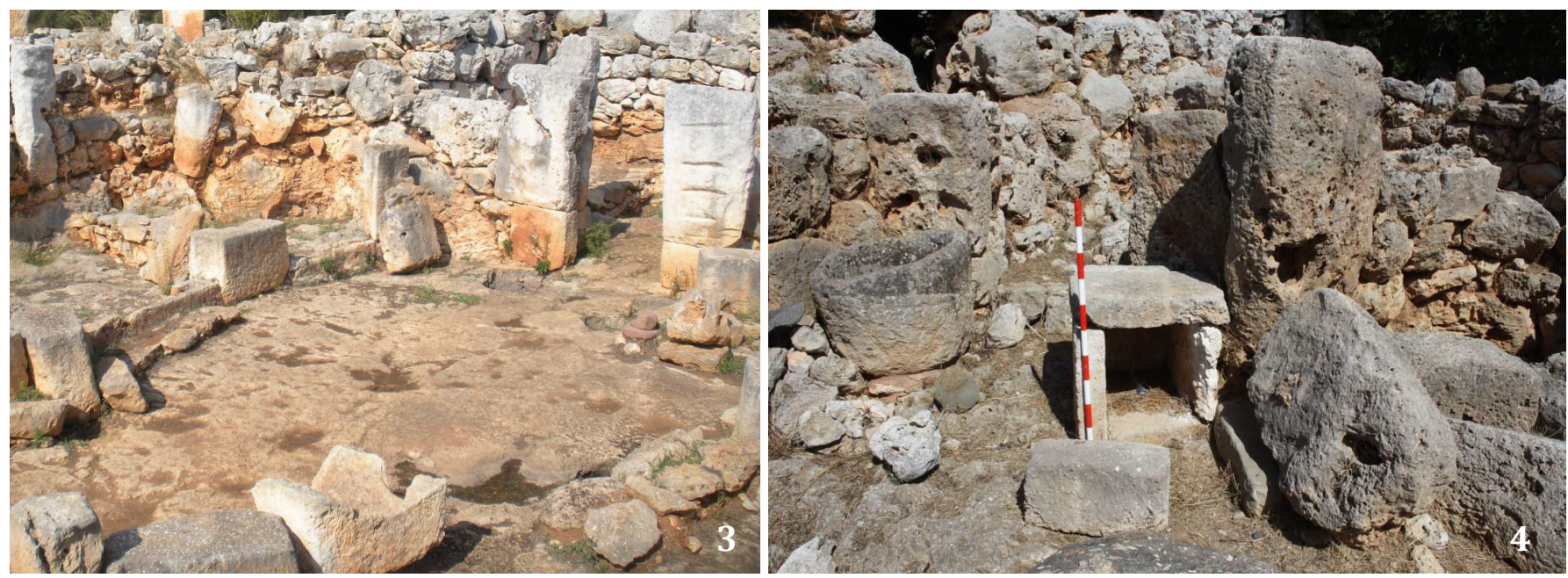

Figs. 3-4. Patio central del Círculo 6 de Torre d'en Galmés. Infraestructura de carácter doméstico en uno de los círculos de Trepucó (Fuente: Proyecto Modular-UA).

un espacio absidal que aloja un hogar. Por su relevante presencia y la abundante cerámica en su entorno inmediato, este espacio se ha interpretado generalmente como un espacio dedicado para la cocción y preparación de alimentos (Serra, 1961; Plantalamor, 1991a; Sintes e Isbert, 2009). La disponibilidad de fuego cercano a la zona del patio, interpretada también como espacio productivo, complementaría las funciones desarrolladas en el mismo.

En cuanto al resto de espacios que componen la casa, encontramos generalmente entre cinco y siete estancias. Una de ellas se ubica inmediatamente en el acceso, y su funcionalidad es un tanto imprecisa, pudiendo dedicarse a almacenamiento o despensa (Ferrer et al., 2011). Por otra parte, dos estancias recurrentes aparecen adosadas entre sí en la parte occidental de la vivienda, de las que destacan especialmente sus umbrales, constituidos por un gran bloque rectangular a soga con entalles acanalados de media profundidad. Asimismo, en la parte oriental se halla un espacio arriñonado que puede aparecer dividido en dos en algunos casos, con funcionalidades aún hoy imprecisas.

La estancia norte está situada, siguiendo el eje de acceso a la vivienda, de forma transversal a la puerta. Es siempre la habitación más grande (Serra, 1961), pero también la más heterogénea. A nivel estructural presenta algunas características diferenciadoras respecto al resto, como su monumentalización mediante diversas fórmulas arquitectónicas (acceso de grandes dimensiones, jambas decoradas, muros interiores de pilastras adosadas, etc.). Ello, unido a otros elementos hallados en diversos círculos (cisternas, cubetas, cultura material, etc.), ha conllevado su lectura generalizada como lugares de cohesión social (Hernández-Gasch, 2011) del propio grupo doméstico residente y de representación pública del mismo con otro tipo de agentes externos.

En cuanto a los yacimientos analizados, se han analizado una gran cantidad de cercles distribuidos en numerosos yacimientos menorquines. Partiendo del que fue principal base empírica para M.L. Serra, Sant Vicenç d'Alcaidús (Alaior) y Biniaiet Vell (Maó) (Serra, 1965; entre muchos otros); pasando por las diferentes zonas de hábitat documentadas en Trepucó (Maó) (Murray, 1932; Plantalamor, 1991; Sintes e Isbert, 2011); el círculo de Torelló (Maó) excavado por Serra (1967); los recintos cubiertos de Talatí de Dalt (Maó) (Juan y Pons, 2005); Sa Torreta de Tramontana (Maó), excavadas también por Murray (1934); Biniparratx Petit (Sant Lluís) (De Nicolás, 1997; Guerrero et al., 2007); el célebre poblado de Torre d'en Galmés (Alaior) con numerosas viviendas excavadas y publicadas 
(Sintes e Isbert, 2009; Ferrer et al., 2011; Lara, 2011; Pérez-Juez et al., 2011; Carbonell, 2012; etc.); Sant Agustí Vell (Es Migjorn Gran) (Plantalamor, 1991); también las viviendas excavadas durante la intervención de urgencia en Ses Talaies de n’Alzina (Ciutadella) (Navarro, 2004); y finalmente el otro gran poblado insular de Son Catlar (Ciutadella) (Juan et al., 1998).

\section{5. ¿UN MODELO DE VIVIENDA POSTALAYÓTICO?}

Hasta ahora se han abordado técnicas de construcción, de materiales empleados y organización espacial. Aparejos, columnas, estancias, patios y hogares son protagonistas indiscutibles, entre muchos otros elementos, de las viviendas postalayóticas baleáricas. Sin embargo, como ha quedado explicitado anteriormente, todo ello conforma una base empírica de estudio que debe servir como cimiento para la construcción de un discurso histórico, es decir, para la interpretación de recurrencias, de ausencias, de elementos que perviven o de aquellos novedosos y/o completamente ajenos.

Una de las principales hipótesis de este trabajo es la apuesta por la existencia de un modelo doméstico propiamente postalayótico. Esto es una forma de construir las casas siguiendo unos patrones concretos que afectan a todo el proceso de erección de la misma, desde los materiales y técnicas empleados hasta el resultado final. Sin embargo, no se trata de un modelo rígido, sino un estándar flexible, adaptable a diferentes condicionantes, pero con patrones transversales de fondo. Ello es posible principalmente por el objeto del modelo: la casa. Las viviendas son construcciones vivas, no diseñadas para perdurar de forma inmutable, sino para adaptarse al ritmo y a la cotidianeidad propia de cada grupo doméstico, en un tiempo y espacio que también está sujeto a cambios.

Que el paso entre Talayótico y Postalayótico cambió por completo el concepto de ámbito doméstico en las Baleares es algo que ya había sido apuntado anteriormente (Lull et al., 2001; Palomar, 2005; Lull et al., 2008; Salvà y Hernández-Gasch, 2009; Hernández-Gasch, 2011; entre otros). Sin embargo, generalmente se ha señalado que el panorama de las viviendas postalayóticas, grosso modo, es notablemente heterogéneo y que está sujeto a variables espacio-temporales, sea en una isla u otra o en función del tramo cronológico concreto del Postalayótico que analicemos (Hernández-Gasch, 2011, pp. 52-53). Por tanto, la propuesta de un modelo que incluya ambas islas, e incluso pueda extrapolarse a la situación observada en Ibiza, es algo completamente nuevo hasta el momento. No sólo por la inclusión de todos los ámbitos domésticos dentro de un mismo estudio, pues existen algunos trabajos sintéticos que abordan el tema de forma transversal (Calvo y Guerrero, 2011), sino por el hecho de proponer unos patrones y esquemas que afecten por igual a las tres islas, aunque Gimnesias y Pitiusas no compartan en origen el mismo devenir histórico y, por ende, tampoco su periodización histórica.

Un rasgo muy característico de este hipotético modelo es el empleo del llamado aparejo ciclópeo. Su empleo no es excluyente, pero sí predominante. Se han detectado otro tipo de aparejos, más simples o complejos, que también forman parte del registro doméstico postalayótico, pero suele tratarse de casos puntuales. Asimismo, se ha documentado en otros ámbitos constructivos el empleo recurrente en este periodo de punteros y cinceles, herramientas que favorecen un mayor trabajo de la piedra y consiguen acabados más escuadrados en los bloques (Prados et al., 2015).

Por otra parte, la morfología general que dibujan esos muros perimetrales de aparejo ciclópeo son en la mayoría de los casos de tendencia rectangular. No se afirma que las plantas rectangulares o cuadradas sean producto únicamente del Postalayótico, pues durante el Talayótico encontramos excelentes ejemplos de este tipo de plantas (Hospitalet Vell, Capocorb Vell, etc.). Sin embargo, en el período que nos ocupa, asistimos a su estandarización. En muchos casos observamos plantas de tendencia rectangular en las que se redondean las esquinas, creando una suerte de planta elíptica. Esto puede interpretarse como una pervivencia transformada de las viviendas arriñonadas del 
Talayótico, como las de S'Illot, pero que ahora adquieren una tendencia más definida. Por otra parte, una gran excepción a este tipo es el panorama menorquín. Los cercles presentan formas en herradura o directamente en círculo. Sin embargo, la presencia de fachadas rectas las hace particulares en sí mismas a nivel morfológico. En cualquier caso, puede establecerse que el modelo de vivienda postalayótico presenta dos variaciones en cuanto a la planta de las casas: tendencias rectangulares y cuadrangulares en Mallorca (con algunas excepciones); y en herradura y circulares en Menorca.

Sin embargo, es la organización espacial la que caracteriza este modelo de hábitat postalayótico. Nos referimos indudablemente a la aparición de un elemento que determina todas y cada una de las viviendas vistas hasta ahora: el patio. Este espacio, generalmente abierto y/o porticado, puede adquirir diversas morfologías, pero en la mayoría de casos ocupa gran parte de la superficie de la casa y cuenta con elementos arquitectónicos concretos que ilustran su morfología descubierta, como las columnas y pilares. La organización de la casa se articula mediante este patio, lo que explica a su vez su distribución espacial. En el caso mallorquín, generalmente encontramos dos estancias en la parte posterior de la casa, adosadas entre sí. Éstas aparecen separadas del resto del edificio por límites arquitectónicos, muros medianeros habitualmente. Sin embargo, en Menorca, generalmente son entre cinco y siete las habitaciones o espacios que podemos encontrar, cada uno delimitado arquitectónicamente y todos dispuestos de forma radial en torno al patio.

\section{6. (IN)CONCLUYENDO: PERSPECTIVAS DE FUTURO}

A modo de síntesis, la lectura que obtenemos de una primera revisión semántica de los datos morfológicos es una cierta homogeneidad en un panorama calificado generalmente como heterogéneo, especialmente entre islas. Bien es cierto que no encontramos el mismo tipo exacto de casas en una isla que en otra, pero ambas responden a un patrón común de hábitat. Sin embargo, como ocurre con todas las generalidades de carácter histórico, cada caso, tiempo y espacio, atiende a una casuística particular a la sazón. Se ha intentado trazar aquí un modelo doméstico caracterizado por diversos patrones, sin anular sus matices y excepciones.

La recurrencia de estas características, adaptadas a los condicionantes del entorno y de los grupos domésticos, hace pensar en una concepción concreta del espacio de hábitat en la mentalidad postalayótica. Los elementos que lo constituyen conforman un esquema interiorizado de vivienda que los grupos domésticos, la sociedad postalayótica en definitiva, crea, asume y reproduce. Es especialmente interesante observar, además, que muchos elementos proceden de tradiciones ajenas a priori a las insulares. Asimismo, la propia existencia de un modelo así ilustraría el profundo cambio que experimentaron las comunidades humanas de las Baleares, pues las casas son probablemente la mejor representación de una cultura, ya que simbolizan sus modos de vida y por ello sus modificaciones son lentas (Rossi, 1977, p. 126).

En definitiva, la propuesta del modelo doméstico postalayótico consiste a la sazón en un conjunto de patrones y recurrencias generado en un espacio y tiempo concreto. Es notablemente flexible, pues sus formas pueden aparecer modificadas en función de los condicionantes del entorno y los propios matices que las comunidades que lo han generado le hayan impreso. Todo ello, en definitiva, engloba una serie de concepciones sociales y culturales (Rapoport, 1969) que se manifiestan a través de la arquitectura doméstica como canalización de todas estas premisas, necesidades y determinaciones sociales (Waisman, 1972). Cabe recordar en este sentido que las viviendas son una parte del enorme engranaje de las sociedades, siendo con seguridad las estructuras definidas en sí mismas que mayor fuerza ejercen como reproductoras de nuevos modelos de vida (Bourdieu, 1972). Por otra parte, los modelos domésticos son herramientas que aglutinan elementos de reconocimiento social, convivencia e hibridación (Gutiérrez, 2012), una suma de características sociales donde se puede reconocer el devenir histórico de las comunidades postalayóticas. 
En un futuro cercano se deberán matizar con mayor precisión las características de este hipotético modelo doméstico, en sus dos principales variantes morfológicas. Las destrucciones de yacimientos característicos durante la primera fase del Postalayótico o la aparición en Mallorca de viviendas complejas en las postrimerías de esta cultura merecen una reflexión de carácter cronológico que permita inferir rasgos evolutivos, diacrónicos, en estos modelos. Es el caso también de las fachadas rectas en las casas menorquinas, no tan comunes en origen, pero progresivamente más habituales; o algunos elementos aparecidos en los patios cuyo uso es aún incierto. Éstas, y el resto de líneas metodológicas propuestas, son las perspectivas de estudio para el futuro inmediato.

\section{BIBLIOGRAFÍA}

Abad Casal, L., Sala Sellés, F. (1993). El poblado ibérico de El Oral. Valencia.

Abad Casal, L., Sala Sellés, F. (2007). La arquitectura y el urbanismo en El Oral (San Fulgencio, Alicante). Un ejemplo de asimilación de la arquitectura fenicia y púnica. En S. Helas y D. Marzoli (Eds.), Phöenizisches und punisches Städtewesen, pp. 499-513.

Aramburu-Zabala, J., Hernández-Gasch, J. (2005). Memoria de las excavaciones arqueológicas en el poblado talayótico de Ses Païsses (Artà-Mallorca). Campañas 1999-2000. Palma de Mallorca.

Belarte, C. (1997). Arquitectura domèstica i estructura social a la Catalunya protohistòrica. Barcelona.

Bermejo Tirado, J. (2009). Leyendolos espacios: una aproximación crítica a la sintaxis espacial como herramienta de análisis arqueológico. Arqueología de la Arquitectura, 6, pp. 47-62.

Blanton, R. E. (1994). Houses and Households. A comparative Study. Interdisciplinary contributions to archaeology. Nueva York.

Bourdieu, P. (1972). Esquisse d'une Théorie de la Pratique. Ginebra.

Calvo, M., Guerero, V. (2011). La cultura Postalayótica (650/550-123 aC). En M. Calvo, A. Aguareles (Eds.), Calvià. Patrimonio cultural, pp. 113-146.

Calvo Trías, M. (2009). Reflexiones en torno a los esquemas de racionalidad espacial reflejados en el paisaje durante la Prehistoria de Mallorca.
PYRENAE, 40 (2), pp 37-78.

Camps, J., Vallespir, A. (1998). Excavacions a Santa Ponça, Mallorca: El Turó de les Abelles. Palma de Mallorca.

Carbonell Salom, M. (2012). El Cercle 7 de Torre d'en Galmés. Estudi d'una estructura domèstica del segle III a.C. a Menorca. Trabajo Fin de Máster. Universidad de Barcelona.

Castro Martínez, P. V., Escoriza Mateu, T., Sanahuja Yll, E. (2003). Mujeres y Hombres en Espacios Domésticos. Trabajo y Vida Social en la Prehistoria de Mallorca (c. 700 - 500 cal ANE). El Edificio Alfa de Puig Morter de Son Ferragut (Sineu, Mallorca). Oxford.

Costa, B., Fernández, J. H. (2006). Historia de las Islas Baleares. Ibiza fenicio-púnica. Palma de Mallorca.

De Nicolàs Mascaró, J. C. (1997). Casa prehistórica en el Aeropuerto de Menorca. Aena Arte, 3, pp. 46-49.

Delgado Hervás, A., Ferrer Martín, M. (2007). Alimentos para los muertos: mujeres, rituales funerarios e identidades coloniales. En P. González, C. Masvidal, S. Montón, M. Picazo (Coord.). Interpreting household practices (Barcelona, 21-24 november 2007), pp. 29-68.

Ferrer, A., Juan, G., Lara, C., Pons, J. (2011). El jaciment de Torre d'en Galmés (Alaior, Menorca). Les intervencions d'Amics del Museu de Menorca: Cercle 7. En J. Gual (Ed.), III Jornades d'Arqueologia de les Illes Balears (Maó, 3 i 4 d'octubre, 2008), pp. 109-117. 
Font Obrador, B. (1970). Excavación en la habitación I. Capocorb Vell. En Actas del XI Congreso Nacional de Arqueología, pp. 415-427.

Frey, O. H. (1968). Zweiter Bericht über die Untersuchungen in der Talayot-siedlung von S'Illot (San Lorenzo, Mallorca). Madrider Mitteilungen 1, 9, pp. 63-75.

Guerrero Ayuso, V. M., Calvo Trías, M., Salvà Simonet, B. (2007). Insularity and the indigenous world on the periphery of the system: the Balearic Islands between the 6th and 1st c. B.C. Journal of Roman Archaeology, 63, pp. 71-84.

Guerrero Ayuso, V. M. (1997). La colonización púnica de Mallorca. La documentación arqueológica y el contexto histórico. Palma de Mallorca.

Guerrero Ayuso, V. M. (2000). Organización del espacio en la factoría púnica de $\mathrm{Na}$ Guardis (Mallorca). En M. Barthélemy, M. E. AubetSemmler (Eds.), Actas del IV Congreso Internacional de Estudios Fenicios y Púnicos, pp. 1539-1554.

Guerrero Ayuso, V. M. (2003). Colonos e indígenas en las Baleares prerromanas. En B. Costa, J. H. Fernández (Eds.), Colonialismo $e$ interacción cultural: el impacto fenicio púnico en las sociedades autóctonas de Occidente, pp. 145203.

Guerrero Ayuso, V. M., Calvo Trías, M., Gornés Hachero, S. (2006). Historia de las Islas Baleares. Mallorca y Menorca en la Edad del Hierro. La cultura Talayótica y Postalayotica. Palma de Mallorca.

Guerrero, V. M., Gornés, S., HernándezGasch, J., De Nicolás, J., Morales, J. V., Morales, A., Pino, B. (2007). Avanç de les investigacions arqueològiques realitzades a l'assentament de Biniparratx Petit (Sant Lluís). En CIME (Eds.), L'arqueologia a Menorca: eina per al coneixement del passat, pp. 9-51.

Gutiérrez Lloret, S. (2012). Gramática de la casa. Perspectivas de análisis arqueológico de los espacios domésticos medievales en la península
Ibérica (siglos VII - XIII). Arqueología de la Arquitectura, 9, pp. 139-164.

Hernández-Gasch, J. (2010). La punta de l'iceberg: reexcavant materials del Fons Colominas (Museu d'Arqueologia de Catalunya). El poblat talaiòtic de Capocorb Vell (Llucmajor, Mallorca). Cypsela, 18, pp. 171-192.

Hernández-Gasch, J. (2011). Privatització i diversificació de l'espai domèstic en la societat balear de l'Edat del Ferro. En J. Gual (Eds.), III Jornades d'Arqueologia de les Illes Balears (Maó, 3 i 4 d'octubre, 2008), pp. 43-62.

Hernández-Gasch, J., Aramburu-Zabala, J. (2005). Murallas de la Edad del Hierro en la Cultura Talayótica. El recinto fortificado del poblado de Ses Païses (Artà, Mallorca). Trabajos de Prehistoria, 62 (2), pp. 125-149.

Hillier, B., Hanson, J. (1984). The social logic of space. Cambridge.

Juan Benejam, Q., Pons Machado, O. (2005). Talatí de Dalt 1997-2001. 5 anys d'investigació a un jaciment talaiòtic tipus de Menorca. Mahón.

Juan Fraile, R., Pons Machado, O., Juan Benejam, Q. (1998). Poblat de Son Catlar. Memòria de la campanya Juny-Setembre de 1995. Ciudadela de Menorca.

Krause, G. (1978). Bericht über die fünfte Granbungskampagne in der Talayotsiedlung von S'Illot (San Lorenzo, Mallorca). Madrider Mitteilungen, 19, pp. 63-75.

Lull Santiago, V., Micó, R., Palomar Puebla, B., Rihuete Herrada, C., Risch, R. (2008). Cerámica Talayótica. La producción alfarera mallorquina entre ca. 900 y 550 antes de nuestra era. Barcelona

Lull Santiago, V., Micó, R., Rihuete Herrada, C., Risch, R. (2001). La prehistoria de les Illes Balears i el jaciment arqueològic de Son Fornés (Montuïri, Mallorca). Montuïri.

Murray, M. (1932). Trapucó. Part I. Cambridge Excavations in Minorca. Londres. 
Murray, M. (1934). Sa Torreta. Cambridge Excavations in Minorca. Londres.

Murray, M. (1938). Trapucó. Part II. Cambridge Excavations in Minorca. Londres.

Navarro Chueca, F. J. (2004). Excavaciones arqueológicas en el yacimiento de "Ses Talaies de n'Alzina". Poblado talayótico afectado por las obras de la ronda Norte (Ciutadella). Mahón.

Palomar Puebla, B. (2005). La ceràmica posttalaiòtica de Mallorca. Significació económica i social dels canvis en el procés productiu entre $c$. 450 - 250 cal ANE. El cas de Son Fornés, Montuïri. Tesis doctoral. Universidad Autónoma de Barcelona.

Pérez-Juez, A. (2011). Excavaciones en la Casa 2 del yacimiento de Torre d'en Galmés, Alaior. Propuestas para el hábitat talayótico. En J. Gual (Ed.), III Jornades d'Arqueologia de les Illes Balears (Maó, 3 i 4 d'octubre, 2008), pp. 119-129.

Plantalamor Massanet, L. (1991). Arquitectura prehistòrica i protohistòrica de Menorca i el seu marc cultural. Mahón.

Prados Martínez, F. (2003). Introducción al estudio de la Arquitectura Púnica. Aspectos formativos y técnicas constructivas. Madrid.

Prados Martínez, F., De Nicolás i Mascaró, J.C., Jiménez Vialás, H., Martínez García, J.J., Torres Gomariz, O. (2015). Culturas arquitectónicas púnicas. Menorca como laboratorio de análisis. En G. Graziani, A. Martínez, J. Escandell (Eds.). VI Jornades d'Arqueologia de les Illes Balears (Formentera, 26 - 28 septiembre), pp. 143-150.

Ramón Torres, J. (2007). Excavaciones arqueológicas en el asentamiento fenicio de "sa Caleta" (Ibiza). Barcelona.

Rapoport, A. (1969). House, form and culture. New Jersey.

Rossi, A. (1977). Arquitectura para los museos. En A. Rossi, F. Serra, J. Romanguera (Coord.). Para una arquitectura de tendencia, pp. 201-210.
Salvà Simonet, B., Hernández-Gasch, J. (2009). Los espacios domésticos en las Islas Baleares durante las Edades del Bronce y del Hierro. De la sociedad Naviforme a la Talayótica. En C. Belarte (Ed.), L'espai domèstic i l'organització de la societat a la protohistòria de la Mediterrània occidental (1er millenni aC): actes de la IV Reunió internacional d'Arqueologia de Calafell, pp. 299322.

Serra Belabre, M. L. (1961). De arqueología menorquina: Círculos. Revista de Archivos, Bibliotecas y Museos, LXIX (2), pp. 951-967.

Serra Belabre, M. L. (1964). Los círculos de Alcaidús: su relación con los monumentos de Malta. En Actas del VIII Congreso Nacional de Arqueología, pp. 243-257.

Serra Belabre, M. L. (1965). Arquitectura ciclópea menorquina. En Arquitectura megalítica y ciclópea catalano-balear, pp. 151-172.

Sintes Olives, E., Isbert Vaquer, F. (2011). El poblado talayótico de Trepucó (Maó, Menorca): pasado y presente de una misma investigación. Informes y Trabajos, 6 (2011), pp. 15-24.

Sintes Olives, E., Isbert Vaquer, F. (2009). Investigación arqueológica y puesta en valor del Recinto Cartailhac. Una unidad doméstica del siglo II ANE en el poblado talayótico de Torre d'en Galmès. Patrimonio Cultural de España, 1, pp. 251-258.

Waisman, M. (1972). La estructura histórica del entorno. Buenos Aires.

Zevi, B. (1978). Il linguagio moderno dell'architettura. Roma. 